\title{
Structural and quantitative evidence of $\alpha 2-6$-sialylated $N$-glycans as markers of the differentiation potential of human mesenchymal stem cells
}

\author{
Kayo Hasehira $^{1,2}$ • Jun Hirabayashi ${ }^{1,2}$ - Hiroaki Tateno ${ }^{1}$
}

Received: 20 April 2016 /Revised: 1 June 2016 / Accepted: 2 June 2016 / Published online: 17 June 2016

(C) The Author(s) 2016. This article is published with open access at Springerlink.com

\begin{abstract}
Human somatic stem cells such as mesenchymal stem cells (hMSCs) have the capacity to differentiate into mesenchymal tissue lineages and to alter immune regulatory functions. As such, they hold promise for use in stem cell-based therapies. However, no method is currently available to evaluate the actual differentiation capacity of hMSCs prior to cell transplantation. Previously, we performed a comprehensive glycan profiling of adipose-derived hMSCs using high-density lectin microarray and demonstrated that $\alpha 2-6$-sialylation is a marker of the differentiation potential of these cells. Nevertheless, no information was available about the structural details of these of $\alpha 2-6$-sialylated glycans. Here we used high performance liquid chromatography (HPLC) analysis combined with mass spectrometry (MS) to perform a structural and quantitative glycome analysis targeting both $\mathrm{N}$ - and $\mathrm{O}$-glycans derived from early (with differentiation ability) and late (without differentiation ability) passages of adipose tissue-derived hMSCs. Findings in these cells were compared with those from human induced pluripotent stem cells (hiPSCs), human dermal fibroblasts (hFibs) and cartilage tissue-derived chondrocytes. A higher percentage of $\alpha 2-6$-sialylated $N$-glycans was detected in early passage cells (24-28\% of sialylated $\mathrm{N}$-glycans) compared with late passage cells (13-15\%). A major $\alpha 2-6$-sialylated $N$-glycan structure detected in adipose-derived hMSCs was that of monosialylated biantennary $\mathrm{N}$-glycan. Similar results were obtained
\end{abstract}

Hiroaki Tateno

h-tateno@aist.go.jp

1 Biotechnology Research Institute for Drug Discovery (BRD), Tsukuba Central 2, 1-1-1 Umezono, Tsukuba, Ibaraki 305-8568, Japan

2 National Institute of Advanced Industrial Science and Technology (AIST), Tsukuba Central 2, 1-1-1 Umezono, Tsukuba,

Ibaraki 305-8568, Japan for the cartilage tissue-derived chondrocytes, Yub621c (28\% for passage 7 and $5 \%$ for passage 28). In contrast, no significant differences were observed between early and late passage hMSCs with respect to $\alpha 2-6$-sialylated $O$-glycan percentages. These results demonstrate that levels of $\alpha 2-6$-sialylated $N$-glycans, but not $O$-glycans, could be used as markers of the differential potential of hMSCs.

Keywords Mesenchymal stem cell · Glycan · Differentiation $\cdot$ Sialylation $\cdot$ Hydrazinolysis

\section{Introduction}

Human mesenchymal stem cells (hMSCs) isolated from tissues such as adipocyte, bone marrow, and umbilical cord blood [1, 2] are attractive cell therapy products (CTPs) for use in regenerative medicine based on their abilities to self-renew and to differentiate into mesenchymal tissue lineages such as osteoblasts, cartilage, and adipocytes [3,4]. This is in addition to their capacity to alter immune regulatory functions [5]. Indeed, hMSCs have already been applied as CTPs to treat patients with diseases such as graft-versus-host disease (GVHD), acute cardiac insufficiency, critical limb ischemia, osteoarthritis, spinal injury, and diabetes $[6,7]$. hMSCs are obtained from the bone marrow or other tissues of donors from different backgrounds in terms of age, sex, race etc. These cells are then expanded in vitro until a required cell number is reached, and are then transplanted into patients following the necessary cell treatment protocols. A complicating factor is that hMSCs are heterogeneous cell populations, whose properties vary depending on the tissues and donors from which they are derived, isolation methods, culture conditions, and culture passages. Therefore, the development of quality control systems with respect to hMSC handling and use is critical to ensure that 
the appropriate therapeutic effects as well as the safety of CTPs is achieved. To this end, no cell surface markers are currently available to evaluate the differentiation potential of stem cells, this being one of the most important measures of the possible therapeutic effects of hMSCs.

To identify cell surface glycan markers that might enable the differentiation potential of hMSCs to be assessed, we build here on glycome analysis work previously carried out on different passages of adipose-derived hMSCs using high-density lectin microarray [8]. We found that $\alpha 2-6$-linked sialic acid $(\alpha 2-$ 6Sia)-specific lectins showed stronger binding to early passage adipose-derived hMSCs with differentiation ability to adipocytes and osteoblasts than did late passage cells without the ability. Flow cytometry analysis also supported the results obtained by lectin microarray. While similar results were obtained for bone marrow-derived hMSCs and cartilage tissue-derived chondrocytes, in the case of human dermal fibroblasts (hFibs), however, which did not have differentiation potential, but did possess a high proliferation ability, no binding of $\alpha 2-6$ Sia-specific lectins was observed. This finding demonstrates that the binding of $\alpha 2-6$ Sia-specific lectins is associated with the differentiation ability of cells, but not to their capacity to proliferate. While this may be the case, no information is currently available concerning the detailed glycan structures of early passage hMSCs (with differentiation ability) compared to late passage cells (without the ability to differentiate).

In this study, we provide structural data for $N$ - and $O$-glycans derived from early and late passages of human adipose tissuederived hMSCs and cartilage tissue-derived chondrocytes. For quantitative comparison, glycans were liberated by gas-phase hydrazinolysis from cells fluorescently tagged with 2aminopyridine at their reducing terminus, following which of the derived pyridylaminated (PA-) glycans were purified by multiple-mode high-performance liquid chromatography (HPLC). The structures of the PA-glycans were determined and quantified by HPLC mapping in conjunction with matrixassisted laser desorption-ionization time-of-flight mass spectrometry (MALDI-TOF MS) and exoglycosidase digestion analyses. We demonstrate that a higher percentage of $\alpha 2-6$ sialylated $\mathrm{N}$-glycans is expressed in early passage hMSCs compared with late passage cells, whereas no significant difference in the percentage of $\alpha 2-6$-sialylated $O$-glycans was observed between early and late passage hMSCs. These results demonstrate that $\alpha 2-6$-sialylated $N$-glycans could serve as markers of the differential potential of hMSCs.

\section{Materials and methods}

\section{Cells}

hMSCs derived from adipose tissues (Life technologies, lot\#: 2117,2118 ), cartilage tissue-derived chondrocytes from polydactylous human fingers (Yub621c, RIKEN CELL BANK, \#: RBRC-HMS0013), and hFibs (ATCC, \#: PCS201-012, Lot: $58,605,481)$ were cultured in $10 \mathrm{~mL}$ of MesenPRO RS ${ }^{\mathrm{TM}}$ Medium (GIBCO, Cat\#: 12,746-012) supplemented with $2 \mathrm{mM} \mathrm{L}$-glutamine and $1 \%$ penicillin-streptomycin on $10 \mathrm{~cm}$ cell culture dishes. Cells were seeded at $3 \mathrm{x}$ $10^{5}$ cells per dish and sub-cultured at $80 \%$ confluency as per the manufacturer's instructions. 201B7 hiPSCs were cultured in $2.5 \mathrm{~mL}$ of mTeSR1 (STEMCELL Technologies) on 6-well plates. Cells were counted with a TC20 Automated Cell Counter (Bio-Rad).

\section{Materials}

Acetonitrile and 1-butanol were obtained from Nacalai Tesque. Acetic acid was purchased from Wako. Dowex 50WX2 (200-400 mesh, $\mathrm{H}^{+}$form) was obtained from Muromachi Technos Co., Ltd. GlycoTAG reagent kit was from Takara Bio. 2, 5-dihydroxybenzoic acid was from Bruker Daltonics. The Sep.-PAK Plus C18 cartridge was from Waters. The Mono Q $5 / 5$ HR column $(5.0 \times 50 \mathrm{~mm})$, the PALPAK Type-R column (4.6 x $250 \mathrm{~mm})$ and the Shodex Asahipak NH2P-50 4D column $(4.6 \times 150 \mathrm{~mm})$ were from GE Healthcare Bio-Sciences Corp, Takara Bio, and Showa Denko, respectively. $\alpha 2-3,-6-$ sialidase (Clostridium perfringens) was from Merck, and $\alpha 2-3$-sialidase cloned from Salmonella typhimurimum LT2 and expressed in Escherichia coli was from Takara Bio. $\beta$-galactosidase, $\beta$ - $N$-acetylhexosaminidase, and $\alpha$-L-fucosidase were from ProZyme, Inc. Standard glycans such as corefucosylated biantennary $N$-glycan (Cat\#: 4109), asialo (Cat\#: 4101), mono- (Cat\#: 4122), di- (Cat\#: 4123), tri- (Cat\#: 4124), and tetra-sialylated $N$-glycans (Cat\#: 4125). were obtained from Takara.

\section{Release of $\mathrm{N}$ - and $\mathrm{O}$-glycans and pyridylamination}

Protein glycans were released from cells by gas-phase hydrazinolysis [9-11] using Hydraclub Y2100 (J-Oil Mills, Inc., Tokyo, Japan). Cells (approximately $1 \times 10^{6}$ cells) were extensively lyophilized in vacuo, and treated with anhydrous hydrazine at $100^{\circ} \mathrm{C}$ for $4 \mathrm{~h}$. After the reaction, the anhydrous hydrazine was removed in vacuo. The released glycans were re- $N$-acetylated with addition of acetic anhydride in a saturated sodium bicarbonate solution, desalted with Dowex 50WX2, and lyophilized [9]. The reducing ends of the liberated glycans were tagged with 2-aminopyridine using GlycoTAG (Takara Bio) and the GlycoTAG reagent kit [12-14]. The bulk of excess reagents in the reaction mixture was removed by phenol/chloroform extraction [15] and subsequent solid-phase extraction using a Sep-PAK Plus C18 cartridge [16]. The PA-glycans were analyzed by HPLC. 


\section{HPLC}

Anion-exchange HPLC was performed on a Mono-Q column at a flow rate of $1.0 \mathrm{ml} / \mathrm{min}$ using two eluents, $\mathrm{A}$ and $\mathrm{B}$. Eluent A was distilled water titrated to $\mathrm{pH} 9.0$ with $1 \mathrm{M}$ aqueous ammonia, and eluent $\mathrm{B}$ was a $0.5 \mathrm{M}$ ammonia acetate solution titrated to $\mathrm{pH}$ 9.0. The column was equilibrated with $100 \%$ eluent A. After injecting a sample, the proportion $(v / v)$ of eluent B was increased linearly to $10 \%$ (3 $\mathrm{min})$, then to $40 \%$ (14 min), and finally to $100 \%$ (5 min). The fluorescence of the eluted PA-glycans was detected with excitation and emission wavelengths of $310 \mathrm{~nm}$ and $380 \mathrm{~nm}$, respectively.

Size-fractionation HPLC was performed on a Shodex Asahipak NH2P-50 4D column $(4.6 \times 150 \mathrm{~mm})$ at a flow rate of $1.0 \mathrm{ml} / \mathrm{min}$ at $25^{\circ} \mathrm{C}$ with a gradient system: two eluents, $\mathrm{C}$ and $\mathrm{D}$, were used, where eluent $\mathrm{C}$ was acetonitrile: water: acetic acid (970:70:3, v/v/v) titrated to $\mathrm{pH} 7.0$ with $7 \mathrm{M}$ aqueous ammonia, and eluent $\mathrm{D}$ was acetonitrile:water:acetic acid (200:800:3, v/v/v) titrated to $\mathrm{pH} 7.0$ with $7 \mathrm{M}$ aqueous ammonia. The column was equilibrated with $95 \%$ eluent $\mathrm{C}$ and $5 \%$ eluent D. After injection of a sample solution, the proportion $(v / v)$ of eluent D was increased linearly from $5 \%$ to $33 \%$ (3 $\mathrm{min}$ ), then to $100 \%$ (75 $\mathrm{min}$ ). Glycan fluorescence was detected at excitation and emission wavelengths of $320 \mathrm{~nm}$ and $400 \mathrm{~nm}$, respectively.

Reversed-phase HPLC was performed on a PALPAK Type-R column (4.6 × $250 \mathrm{~mm}$ ) with two eluents, E and F, where eluent $\mathrm{E}$ was $100 \mathrm{mM}$ ammonium acetate, $\mathrm{pH} 4.0$, and eluent $\mathrm{F}$ was $100 \mathrm{mM}$ ammonium acetate, $\mathrm{pH} 4.0$, containing $0.5 \%(v / v) 1$-butanol. The column was equilibrated with $75 \%$ eluent $\mathrm{E}$ and $25 \%$ eluent $\mathrm{F}$. After injection of a sample, the proportion $(v / v)$ of eluent $\mathrm{F}$ was increased linearly from $25 \%$ to $100 \%(60 \mathrm{~min})$ at a flow rate of $1.0 \mathrm{ml} / \mathrm{min}$ at $25{ }^{\circ} \mathrm{C}$. Glycan fluorescence was detected at excitation and emission wavelengths of $320 \mathrm{~nm}$ and $400 \mathrm{~nm}$, respectively.

\section{MS}

The purified PA-glycans were subjected to analysis by MALDI-TOF MS with an Ultraflex mass spectrometer (Bruker Daltonics, Bremen, Germany), equipped with a 337$\mathrm{nm}$ nitrogen laser, and set at $20 \mathrm{kV}$ extraction voltage. 2, 5dihydroxybenzoic acid ( $1 \mathrm{mg} / \mathrm{ml}$ in $30 \%$ ethanol) was used as the matrix. Analyses were carried out in reflector mode over a mass range of $\mathrm{m} / \mathrm{z} 0$ to 2000 , or 1000 to 4000 in the positive ion mode. Each spectrum was measured by 150 laser shots.

\section{Quantification of PA-saccharides}

Each PA-glycan was quantified by the peak area compared with that corresponding to an appropriate authentic standard separated under the same HPLC conditions. PA-GlcNAc was used as the authentic calibration standard. Relative yields were expressed as percentages compared to the total amounts of $N$ linked or $O$-linked glycans prepared from each of the cell samples.

\section{Exoglycosidase treatment}

Exoglycosidase treatment of PA-glycans was carried out by using $\alpha 2-3,-6$-sialidase (C. perfringens: Merck), $\alpha 2-3-$ sialidase ( $S$. typhimurimum LT2, recombinant, E. coli: Takara Bio), $\beta$-galactosidase (Jack bean: ProZyme, Inc.), $\beta$ $N$-acetylhexosaminidase (Jack bean: ProZyme, Inc.), or $\alpha$-Lfucosidase (bovine kidney: ProZyme, Inc.). PA-glycan was treated with the enzyme $(50 \mathrm{mU})$ in $20 \mu \mathrm{l}$ of $50 \mathrm{mM}$ ammonium acetate buffer ( $\beta$-galactosidase: $\mathrm{pH} 3.5, \alpha 2-3,-6$ sialidase and $\beta$ - $N$-acetylhexosaminidase: $\mathrm{pH} 5.0, \alpha 2-3$ sialidase and $\alpha$-L-fucosidase: $\mathrm{pH} 5.5)$, at $37^{\circ} \mathrm{C}$ for appropriate periods $(0.5 \mathrm{~h}$ for $\alpha 2-3,-6$-sialidase and $\alpha 2-3$ sialidase; $20 \mathrm{~h}$ for others). The enzyme reaction was terminated by heating at $99.9^{\circ} \mathrm{C}$ for $5 \mathrm{~min}$.

\section{Analysis of the Sia linkage type}

The sialic acid linkage type was determined by its sensitivity to $\alpha 2-3,-6$-sialidase (C. perfringens, Merck) and $\alpha 2-3$ sialidase (S. typhimurimum LT2, recombinant, E. coli, Takara Bio) treatment. Each acidic saccharide was treated with both sialidases, and the products were analyzed by anion-exchange HPLC using a Mono-Q column. If an acidic glycan has the $\alpha 2-3$ type of sialic acid linkage, its retention time should decrease after digestion both with $\alpha 2-3,-6$ and $\alpha 2-3$-sialidases. In contrast, if the glycan has the $\alpha 2-6$ type linkage, its retention time should be preserved after $\alpha 2-3$ sialidase treatment. When all Sia residues are removed by the action of $\alpha 2-3,-6$-sialidase, a neutral glycan should be generated.

\section{Results}

\section{Basic strategy of glycan analysis of adipose-derived hMSCs}

$\mathrm{N}$ - and $\mathrm{O}$-glycans were liberated by gas-phase hydrazinolysis from early and late passages of lyophilized adipose-derived hMSCs. Two lots of early and late passages of adipose-derived hMSCs (lot\#: 2117, passage 5 (P5) and P26; lot\#: 2118, P3 and P28) were analyzed to confirm the reproducibility and consistency of the results. Whole glycans comprising both intracellular (ER and Golgi) and surface membranous fractions were re- $N$-acetylated and labeled with 2-aminopyridine.

The derived PA-glycans were first separated by anionexchange HPLC (Fig. 1a). Neutral glycans, which passed 
a

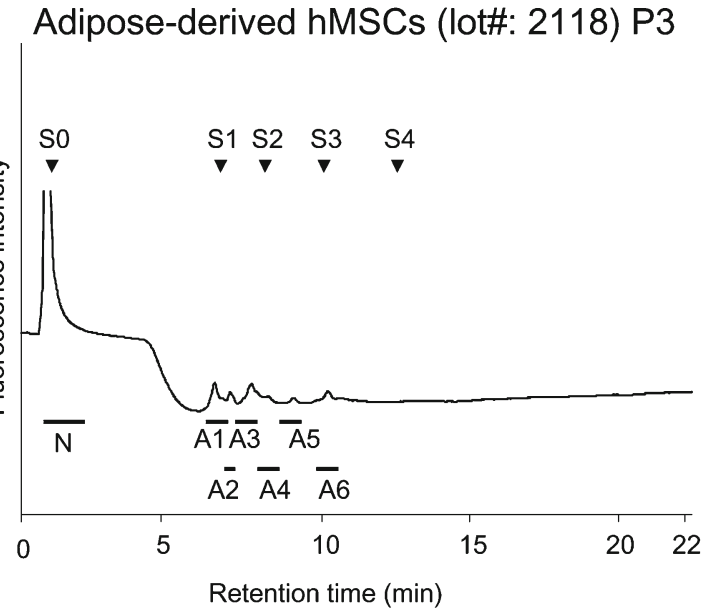

b
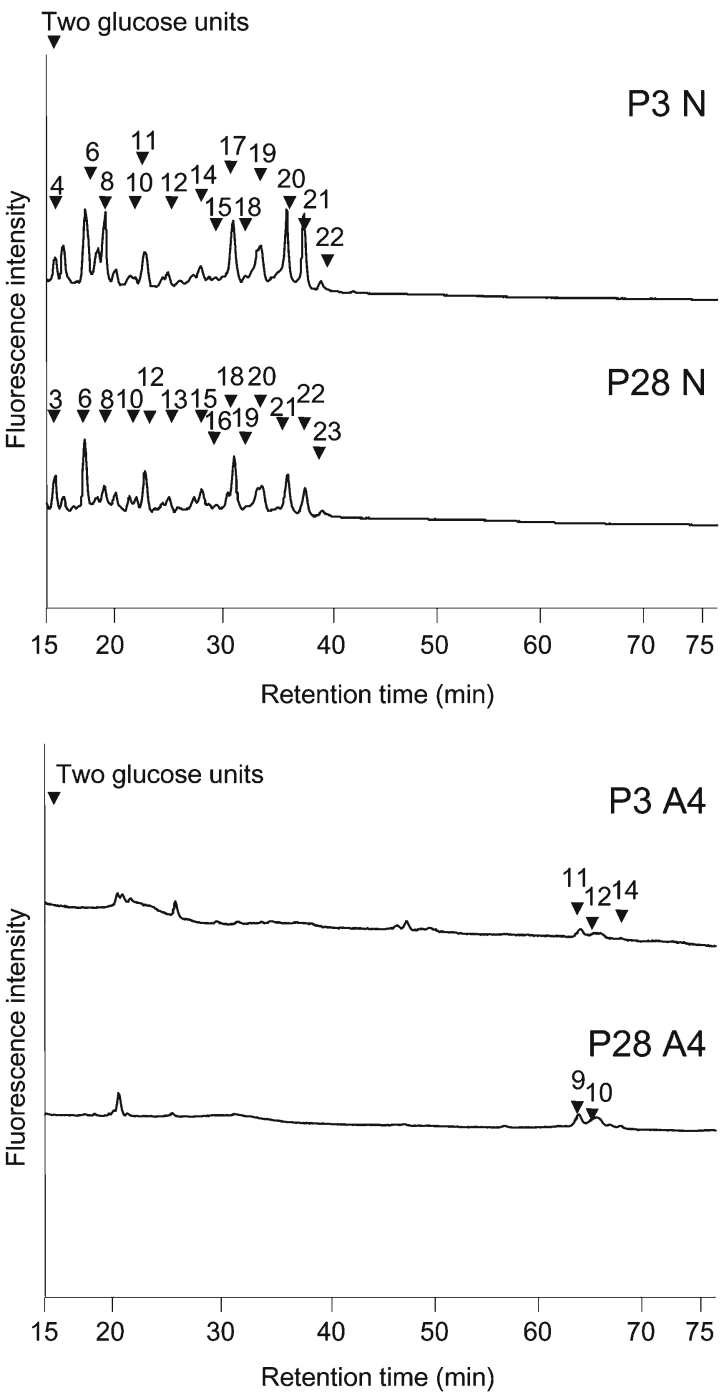

Adipose-derived hMSCs (lot\#: 2118) P28
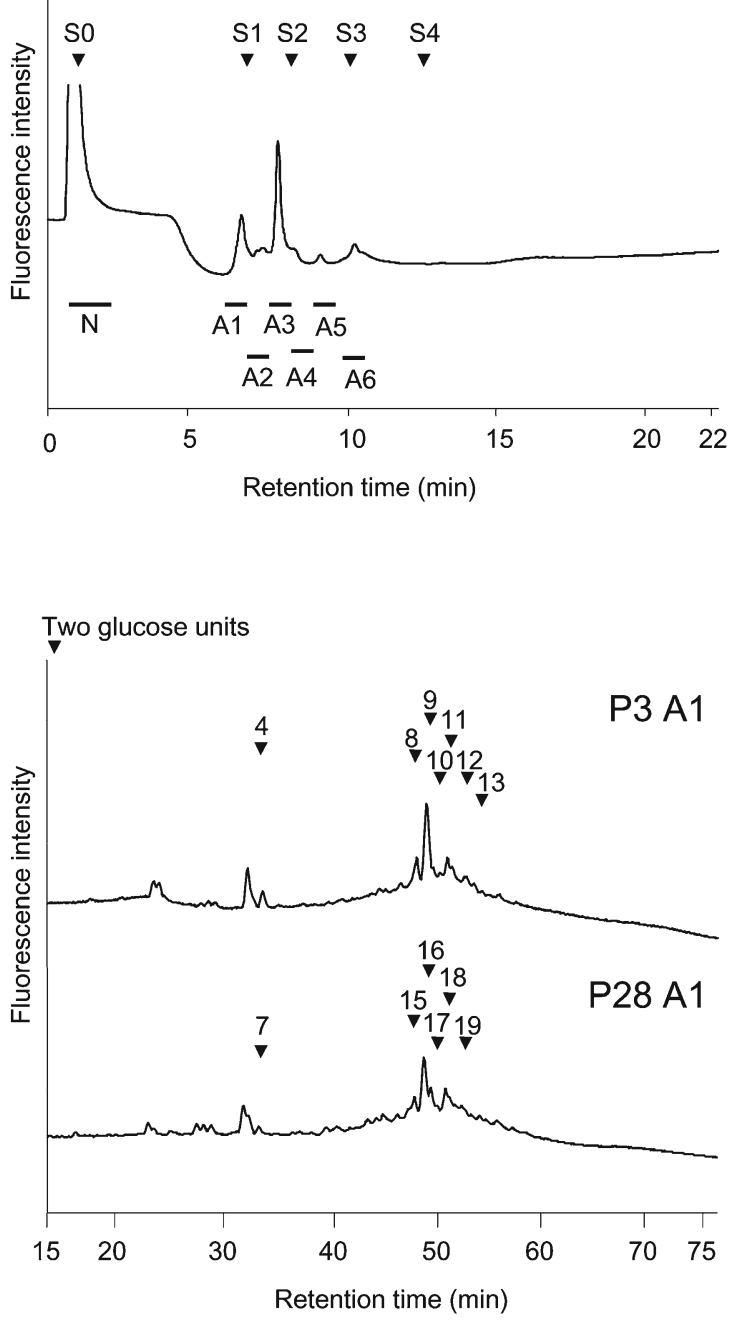
Fig. 1 Comparison of HPLC profiles of glycans derived from adiposederived hMSCs lot\#: 2118 P3 and P28. a Anion-exchange HPLC profiles. S0, S1, S2, and S3 are standard $N$-glycans containing $0,1,2$, and 3 Sia residues. b Size-fractionation HPLC profiles of neutral fraction (N), monosialylated $N$-glycan fraction (A1), and disialylated $N$-glycan fraction (A4). Following anion-exchange HPLC, further purification was performed. Each peak was pooled, and when necessary, was further purified by reversed-phase HPLC, or subjected to further structural analysis. Arrowheads indicate the peak numbers

through the column, were pooled and designated the " $\mathrm{N}$ " fraction. Adsorbed anionic glycans were fractionated and designated the "A1-A6" fractions (according to the order of elution, Fig. 1a). These fractions were then subjected to sizefractionation HPLC (for fractions N, A1 and A4, see Fig. 1b).
Each peak was fractioned, and when necessary, the eluate was further purified by reversed-phase HPLC (chromatogram not shown). Each purified PA-glycan was quantified on the basis of fluorescence intensity relative to an appropriate authentic standard (PA-GlcNAc).

The purified neutral glycans (No.1-16 and 28-29) were analyzed by MALDI-TOF MS and reversed-phase HPLC (chromatogram not shown), and when necessary, were treated with glycosidases to confirm their structure. As a result, unambiguous assignment was made for all glycans, with $16 \mathrm{~N}$ glycans and $2 \mathrm{O}$-glycans identified as neutral glycans in four different cell lines (Tables 1 and 2).

Acidic glycans (A1-A6 fractions) were analyzed by a combination of sialidase digestion, MALDI-TOF MS and

Table 1 List of $N$-glycans identified in adipose-derived hMSCs

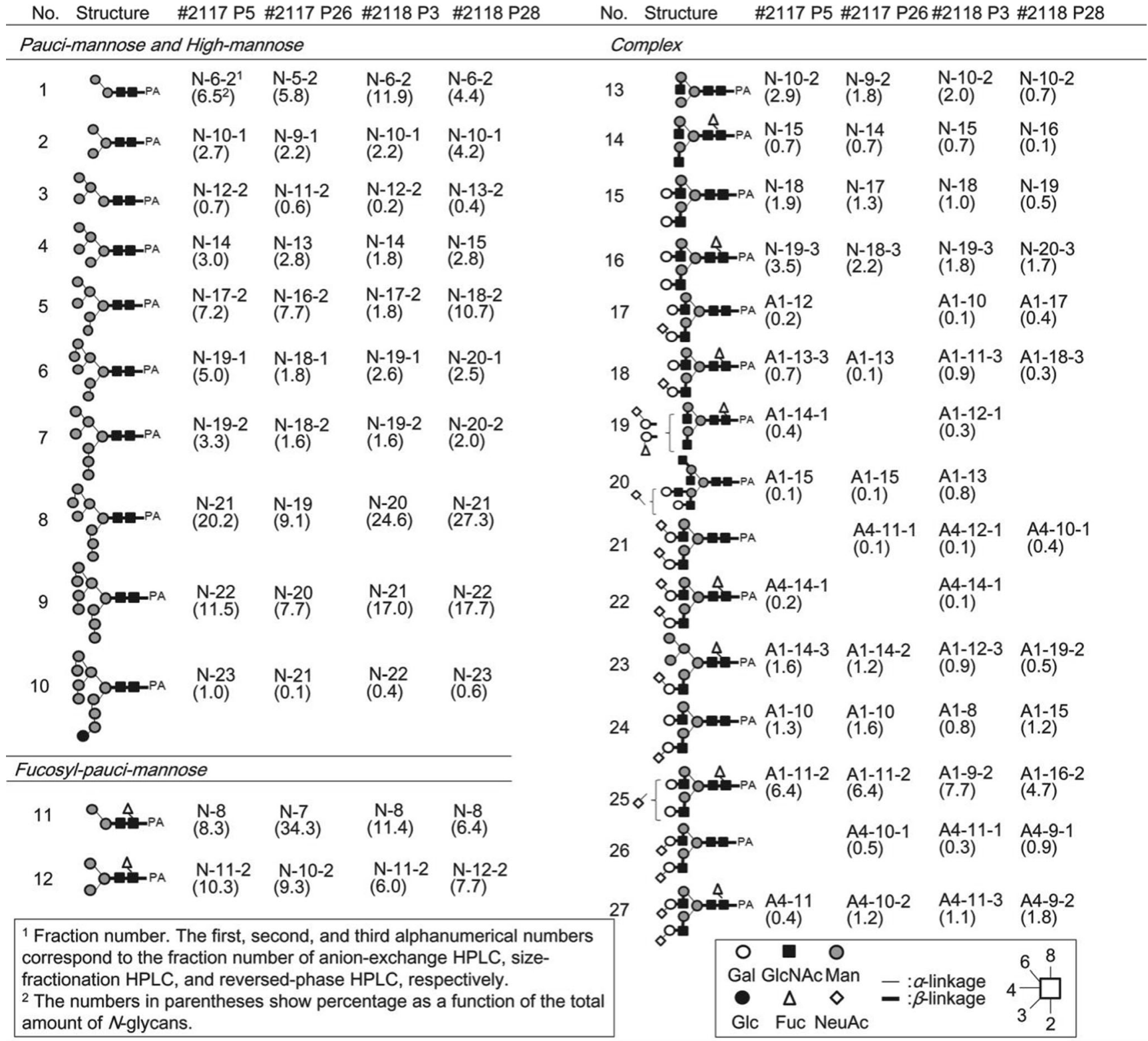


Table 2 List of $O$-glycans identified in adipose-derived hMSCs

No. Structure \#2117 P5 \#2117 P26 \#2118 P3 \#2118 P28

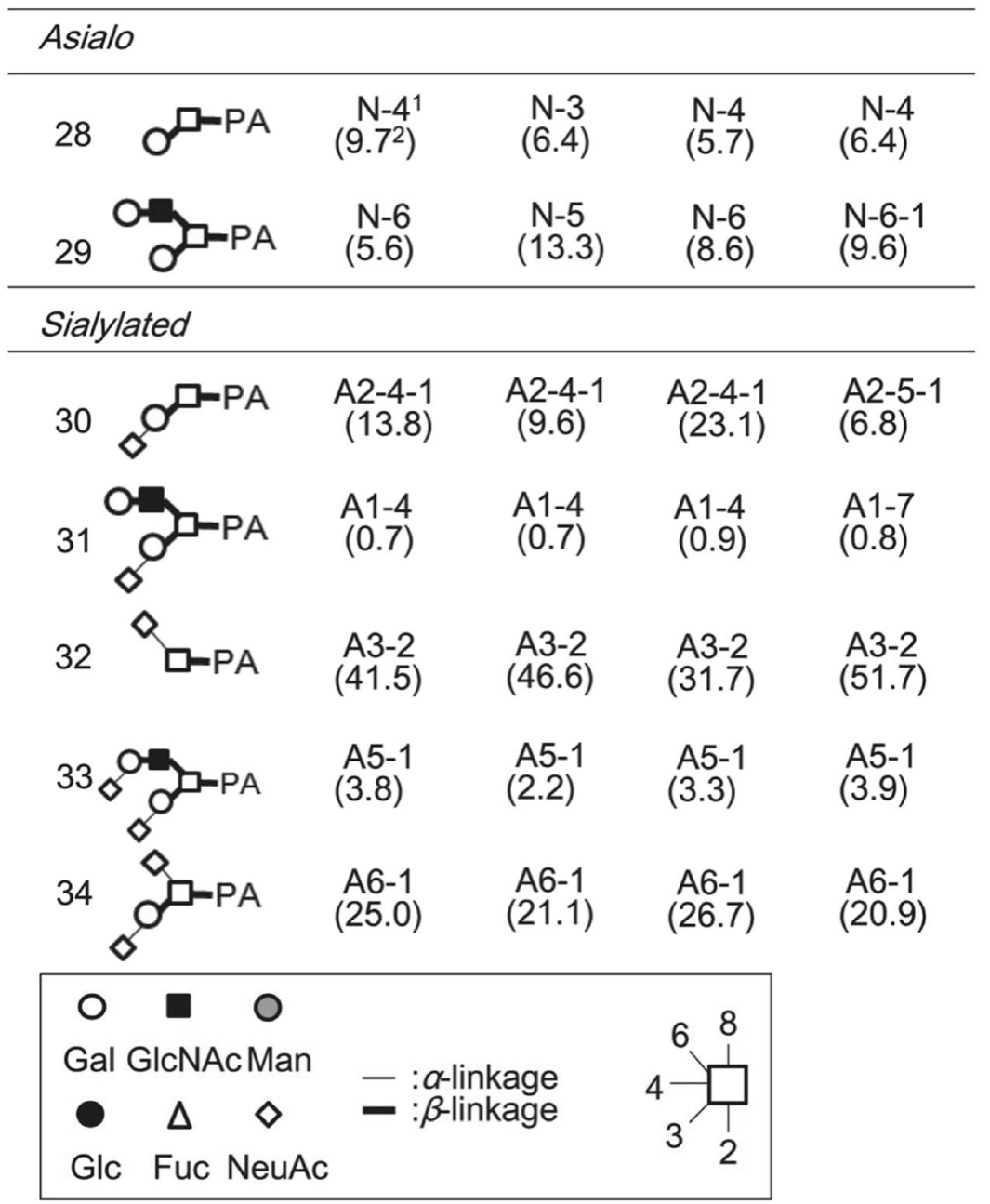

\footnotetext{
${ }^{1}$ Fraction number. The first, second, and third alphanumerical numbers correspond to the fraction number of anion-exchange HPLC, size-fractionation HPLC, and reversed-phase HPLC, respectively.

The numbers in parenthesis show percentage as a function of the total amount of $O$-glycans.
}

reversed-phase HPLC. After digestion with universal $\alpha 2-3,-6$-sialidase, the products were passed through a Mono-Q column, analyzed by MALDI-TOF MS and reversed-phase HPLC. Their structures were determined as described above. The type of linkage of each sialic acid was determined by susceptibility to two sialidases, i.e., $\alpha 2-3$-specific sialidase and the universal $\alpha 2-3,-6$-sialidase. For example, the structure of $\alpha 2-6$-disialylated, core-fucosylated biantennary $N$-glycan, A4-14-1, derived from adiposederived hMSCs (Lot\#:2117 P5) was determined as follows. When A4-14-1 was treated with $\alpha 2-3,-6$-sialidase (C. perfringens, Merck) and analyzed by MALDI-TOF MS, the glycan was determined as the monofucosylated biantennary glycan $\left(\mathrm{m} / z, 1865.5[\mathrm{M}+\mathrm{H}]^{+}\right.$in positive mode), which exactly matched with the elution time of the standard core-fucosylated biantennary $\mathrm{N}$-glycan by reversed-phase HPLC. On the other hand, when A4-14-1 was treated with $\alpha 2-3$-sialidase, no Sia was released, demonstrating that the linkage mode of Sia of A4-14-1 is $\alpha 2-6$ Sia. Disialylation on both branches of biantennary $\mathrm{N}$-glycans could be confirmed by comparing with the retention time with standard glycans of asialo, mono-, di-, tri-, and tetra-sialylated $\mathrm{N}$-glycans in an anion-exchange chromatography.

As a result, 9 and 8 acidic $N$-glycans were identified from P5 and P26 of adipose-derived hMSCs (lot\#: 2117), respectively, while 11 and 8 acidic $N$-glycans were identified from lot\#: 2118 P3 and P28, respectively (Table 1). Five acidic $O$ glycans were identified in A1-6 fractions obtained from four different cell lines (Table 2). Finally, 25, 24, 27, and $24 \mathrm{~N}$-glycans were identified from P5 and P26 of adipose-derived hMSCs from lot\#: 2117, and P3 and P28 of adipose-derived hMSCs from lot\#: 2118 , respectively (Table 1). Seven $O$-glycan structures were identified from the cell lines (Table 2). 

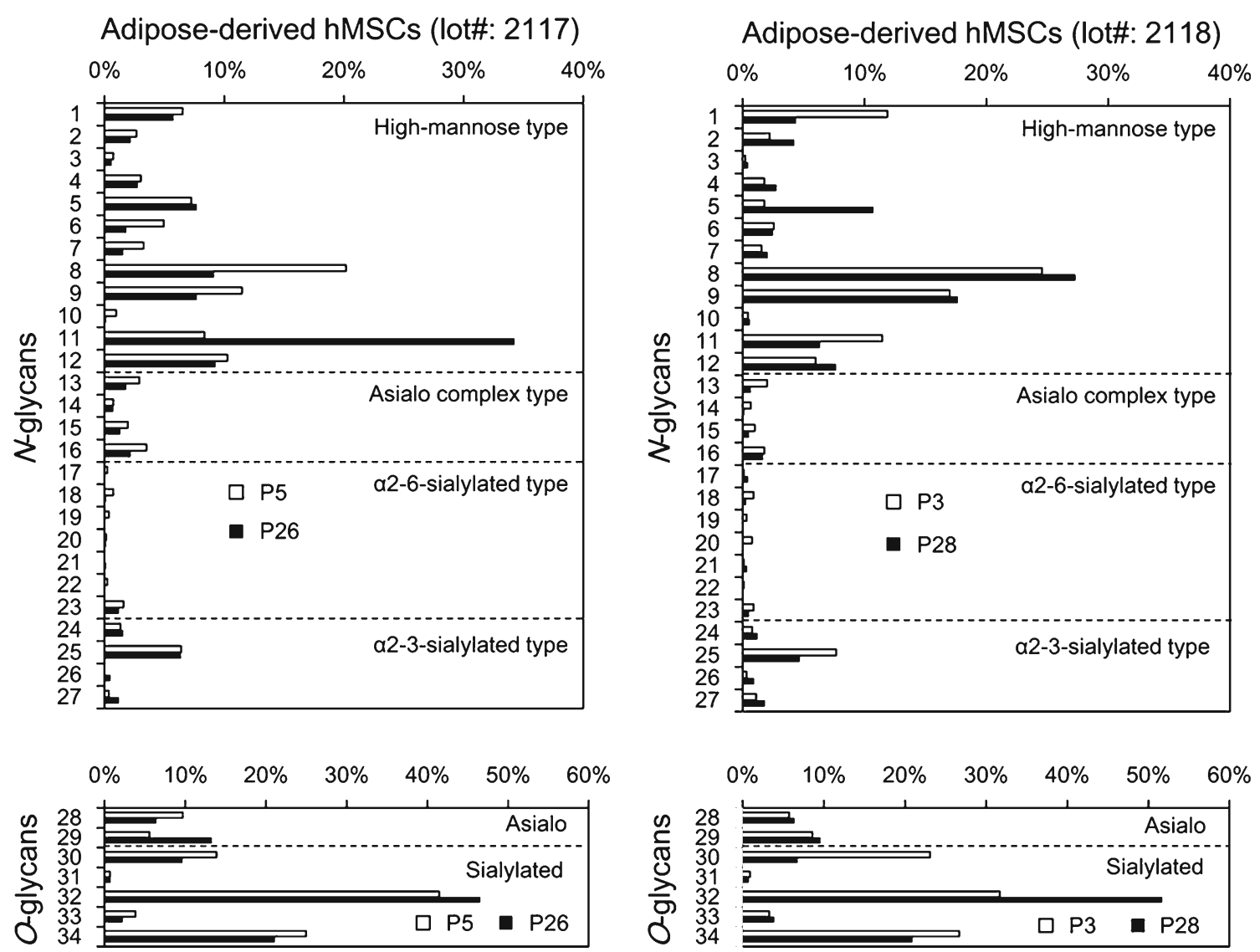

Fig. 2 Comparison of $N$ - and $O$-glycans prepared from adipose-derived hMSCs lot\#: 2117 and lot\#: 2118. Each glycan is expressed as a percentage of the total amount of $\mathrm{N}$ - or $\mathrm{O}$-glycans obtained from each sample (taken as $100 \%$ ). Glycan numbers correspond to those used in Tables 1 and 2

\section{Comparative analysis of $N$ - and $\boldsymbol{O}$-glycans between early and late passages of adipose-derived hMSCs}

We next compared the percentage of each glycan derived from early and late passages of adipose-derived hMSCs, taking the total amounts of $\mathrm{N}$ - or $\mathrm{O}$-glycans prepared from each sample to be $100 \%$ (Fig. 2). Among $N$-glycans, high-mannose type $N$-glycans (No.1-12) accounted for a substantial fraction in both the early and late passages of these cells. Interestingly, the expression patterns of high-mannose type $N$-glycans were significantly different between the two lots. In terms of No. 8 glycan, for example, early passage cells (P5) of adiposederived hMSCs (lot\#: 2117) exhibited higher percentage than late passage cells (P26), whereas late passage cells (P28) gave higher percentage than early passage cells (P3) in lot\#: 2118. In terms of No. 11 glycan, late passage cells (P26) exhibited much higher percentage than early passage cells (P5) of adipose-derived hMSCs (lot\#: 2117), whereas early passage cells (P3) gave higher percentage than late passage cells (P28) in lot\#: 2118.

Asialo complex type (No.13-16) and sialylated $N$-glycans (No. 17-27) were also detected in significant amounts. A hybrid type $N$-glycan was also observed (No. 23) (Table 1). Among the $O$-glycans, interestingly, a high percentage of sialylated $O$-glycans was detected in adipose-derived hMSCs (Fig. 3). Sialyl Tn (No. 32) gave the highest percentage in both early and late passages of adipose-derived hMSCs (Fig. 2 and Table 2). Disialyl T (Sia $\alpha 2-3$ Gal $\beta 1-$ 3(Sia $\alpha 2-6)$ GalNAc, No. 34) was also detected in significant amounts. Core1 (Gal $\beta 1-3 \mathrm{GalNAc}$, No. 28) and core2 [Gal $\beta 1-4 G l c N A c \beta 1-6(G a l \beta 1-3) G a l N A c$, No. 29], and their sialylated forms (No. 30,31, 33, 34) were also detected. The percentage of sialyl Tn (Sia $\alpha 2-6$ GalNAc, No. 32) was observed to be higher in late passage cells (lot\#: 2117 P26 and lot\#: 2118 P28) compared with early passage cells (lot\#: 2117 P5 and lot\#: 2118 P3).

\section{Comparative analysis of $N$ - and $O$-glycans of adipose-derived hMSCs with other cell types}

Overall signatures of $\mathrm{N}$ - and $\mathrm{O}$-glycans of adipose-derived hMSCs were compared to hiPSCs (cell line 201B7) as a representative of human pluripotent stem cells, and to hFibs as a representative of somatic cells, in addition to another type of somatic stem cell, cartilage tissue-derived chondrocytes (Yub621c, P7 and P28) (Fig. 3). All of the cells gave similar patterns of $N$-glycan types. As described above, highmannose type $N$-glycans gave the highest percentage among 
the types of $N$-glycans that were present. Asialo and sialylated $N$-glycans were also observed.

In contrast, the overall $O$-glycan signatures were different depending on the cell types under consideration. Interestingly, adipose-derived hMSCs gave a high percentage of sialylated $O$-glycans (average $84 \%$ ) relative to hiPSCs (39\%), hFibs (59\%), and cartilage-derived chondrocytes $(31 \%)$.

\section{$\alpha 2-6$-sialylated $\mathrm{N}$-glycans, but not $\mathrm{O}$-glycans, are markers of the differentiation potential of stem cells}

We subsequently focused our attention on sialylation, since $\alpha 2-6$ Sia-binding lectins showed stronger binding to early passage hMSCs derived from adipocytes and bone marrow than their corresponding late passage cells, as previously reported [8]. Figure 4 summarizes the Sia linkage type in $N$ - and $O$ glycans, where " $\alpha 2-3 \mathrm{Sia}$ " and " $\alpha 2-6 \mathrm{Sia}$ " indicate $N$ - or $O$ glycans containing $\alpha 2-3 \mathrm{Sia}$ and $\alpha 2-6 \mathrm{Sia}$, respectively, and " $\alpha 2-3 \mathrm{Sia}+\alpha 2-6 \mathrm{Sia}$ " indicate $N$ - or $O$-glycans containing both $\alpha 2-3$ Sia and $\alpha 2-6$ Sia.

In terms of $\mathrm{N}$-glycans, 201B7 hiPSCs expressed exclusively $\alpha 2-6 \mathrm{Sia}(100 \%)$, whereas, in contrast, hFibs expressed mostly $\alpha 2-3 \mathrm{Sia}$ ( $1 \%$ for $\alpha 2-6 \mathrm{Sia}$ ) [17]. In agreement with the previous report [8], the percentage of $\alpha 2-6$-sialylated $N$ glycans (Fig. 4, black and grey) was indeed higher in early passage adipose-derived hMSCs (29\% for lot\#: 2117 P5,
$25 \%$ for lot\#: 2118 P3) than for corresponding late passage cells (14 \% for lot\#: 2117 P26, $16 \%$ for lot\#: 2118 P28). Similarly, early passage cartilage tissue-derived chondrocytes ( $29 \%$ for P7) expressed a higher percentage of $\alpha 2-6$ sialylated $N$-glycans than corresponding late passage cells (5\% for P28). A major $\alpha 2-6$-sialylated $N$-glycan structure detected in adipose-derived hMSCs and cartilage tissuederived chondrocytes was "mono-sialylated biantennary $\mathrm{N}$ glycan" (Fig. 2 and Table 1). $O$-glycans containing $\alpha 2-6 \mathrm{Sia}$ such as sialyl Tn (Sia $\alpha 2-6$ GalNAc) and disialyl T (Sia $\alpha 2-$ 3Gal $\beta 1-3(\operatorname{Sia} \alpha 2-6)$ GalNAc) were also detected in $O$-glycans (Table 2). However, no significant relationship was observed between the differentiation potential of stem cells and the Sia linkage mode of $O$-glycans. Taken together, these results clearly demonstrate that $\alpha 2-6$-sialylated $N$-glycans, but not $O$-glycans, are markers of the differentiation potential of stem cells.

\section{Discussion}

Previously, we performed a quantitative glycome analysis targeting both $N$ - and $O$-glycans derived from 201B7 hiPSCs and hFibs representing undifferentiated and differentiated cells, respectively, using the same strategy described in the present report [17]. A dramatic glycome shift became
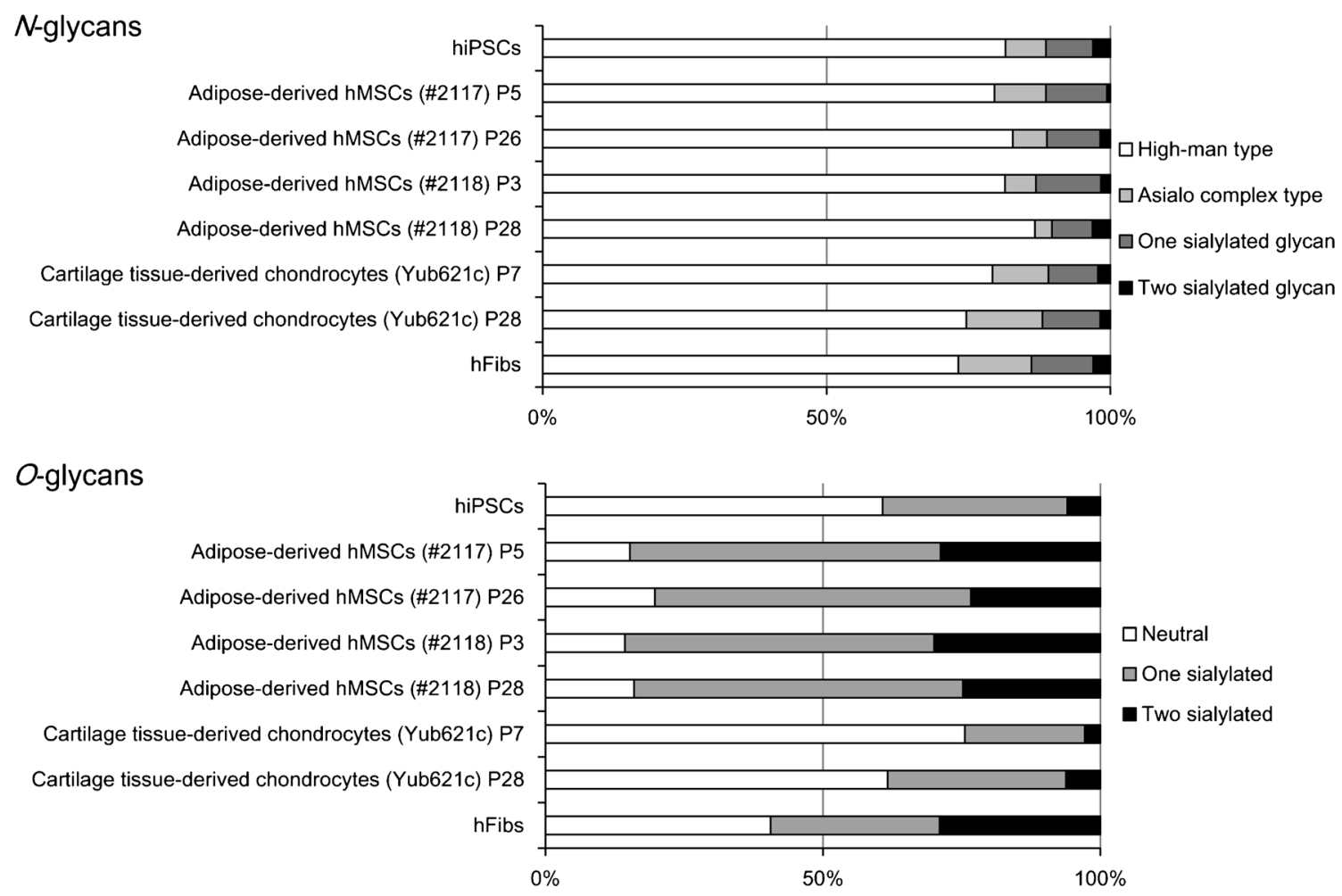

Fig. 3 Summary of glycan type. Comparison of the glycan type and proportion in $\mathrm{N}$ - and $\mathrm{O}$-glycans prepared from hiPSCs, adipose-derived hMSCs (lot\#: 2117) P5 and P26, adipose-derived hMSCs (lot\#: 2118) P3

and P28, cartilage tissue-derived chondrocytes (Yub621c) P7 and P28, and hFibs. Results were calculated based on the data in Tables 1 and 2 
evident upon conversion from differentiated hFibs to undifferentiated hiPSCs. One of the most significant changes was the Sia linkage mode, which for $N$-glycans of 201B7 hiPSCs was found to consist exclusively of $\alpha 2-6$ Sia, whereas that of hFibs was mostly of the $\alpha 2-3$ Sia type [17]. Recently, using the systematic glycan profiling system called high-density lectin microarray, we found that $\alpha 2-6$ Sia-specific lectins (SNA, SSA, TJA1, and rPSL1a) show stronger binding to early passage cells (with differentiation ability) than late passage cells (without this ability) [8]. Similar results were observed for bone marrow-derived hMSCs and cartilage tissue-derived chondrocytes. Furthermore, the removal of Sia by sialidase treatment significantly reduced the differentiation efficiency of hMSCs. Therefore, we proposed that $\alpha 2-6$-sialylation could be a functional marker of the differentiation potential of stem cells. In the present report, we have performed a structural and quantitative analysis of the glycome of early and late passages of adipose- and cartilage tissue-derived chondrocytes using HPLC analysis combined with MS. We clearly demonstrate that the percentage of $\alpha 2-6 \mathrm{Sia}$-containing $N$-glycans, but not $O$-glycans, was found to be higher in early passage cells than late passage cells. Therefore, $\alpha 2-6$-sialylaed $N$-glycans could serve as markers of the differentiation potential of stem cells.
SNA and SSA, but not TJA1 and rPSL1a, bound to bovine submaxillary mucins expressing sTn as described in the previous report [8]. Therefore, sTn could be target glycans for SNA and SSA, although sTn showed no relationship with the differentiation capacity of hMSC. In this sense, TJA1 and rPSL1a without the binding affinity to sTn might be better probes for the purpose of the evaluation of the differentiation capacity of hMSCs.

The expression of $\alpha 2-6$-sialyltransferase (ST6Gal-I) has been shown to play an important role in the regulation of cellular pluripotency in human pluripotent stem cells [18-20]. Therefore, the key phenomena might be the changes of the expression of ST6Gal-I. ST6Gal-I catalyzes the addition of terminal $\alpha 2-6$ Sia to $N$-glycans, but not $O$-glycans. This might be the reason why $\alpha 2-6$-sialylation on $N$-glycans, but not $O$-glycans, changes depending on the differentiation potential of hMSCs. Combined with our findings showing that $\alpha 2-6 \mathrm{Sia}$ is dominant on $N$-glycans of hiPSCs and correlates with the differentiation capacity of cells, it seems that the expression of $\alpha 2-6$-sialylated $N$-glycans is associated with "stemness".

What are the roles of $\alpha 2-6$ Sia in stem cell functions? Previously we showed that $\alpha 5$ integrin with $14 \mathrm{~N}$-glycosylation

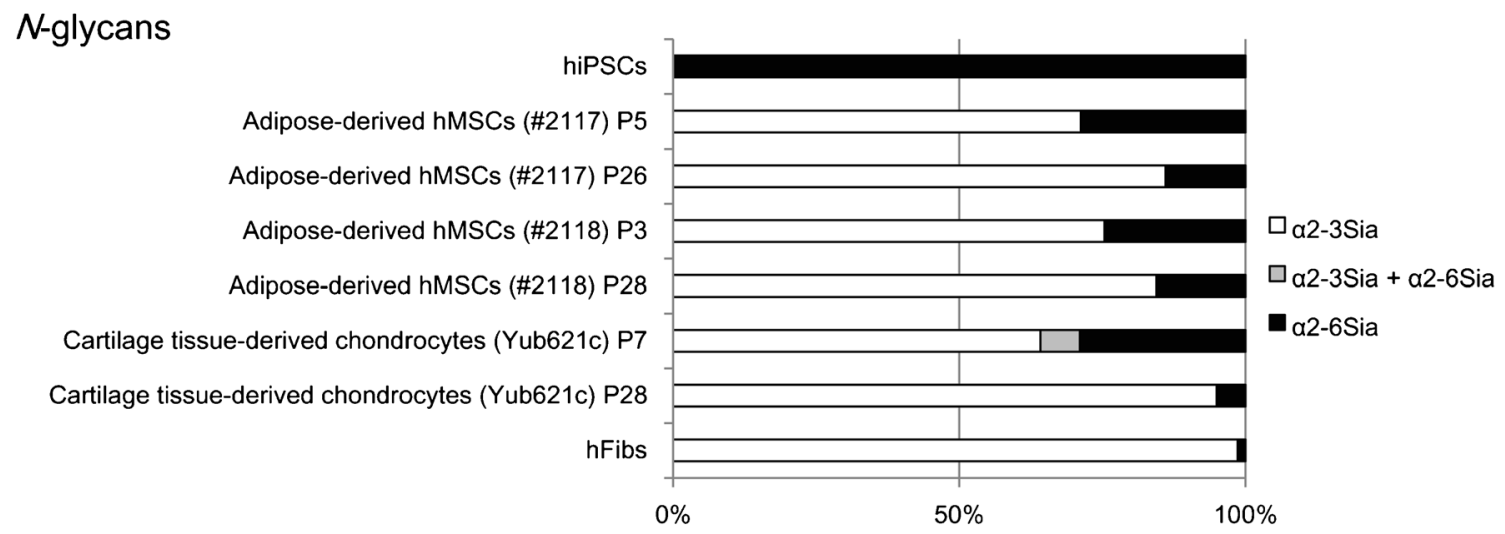

O-glycans

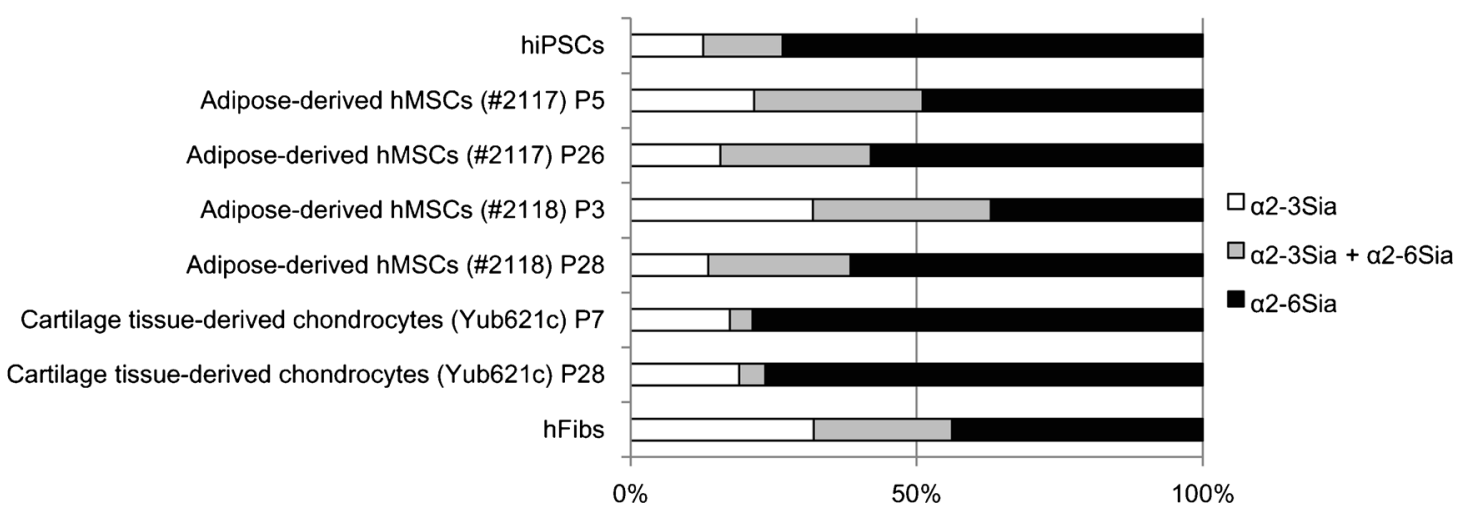

Fig. 4 Linkage mode of Sia. Comparison of the Sia linkage type in $N$-and $O$-glycans prepared from hiPSCs, adipose-derived hMSCs (lot\#: 2117) P5 and P26, adipose-derived hMSCs (lot\#: 2118) P3 and P28, cartilage tissuederived chondrocytes (Yub621c) P7 and P28, and hFibs. Data are

expressed as a percentage of the total amount of sialylated $N$ - or $O$-glycans obtained from each sample (taken as $100 \%$ ). Results were calculated based on the data provided in Tables 1 and 2 
sites is one of the carrier proteins of $\alpha 2-6$ Sia in cartilage tissuederived chondrocytes [8]. $\alpha 5 \beta$ 1integrin is a well-known fibronectin receptor, and its interaction with fibronectin is essential for cell migration and proliferation, for the activation of intracellular signaling pathways, and cytoskeletal formation [21]. Therefore, $\alpha 2-6$ Sia on $\alpha 5$ integrin could modulate interactions with matrix components such as fibronectin and regulate the differentiation potency of stem cells. Further studies are required to understand the roles of $\alpha 2-6 \mathrm{Sia}$ in this process.

The development of novel markers to evaluate the properties of hMSCs is keenly required for the realization of safe stem cell-based therapy, with such cells being used as hCTPs in regenerative medicine to treat various diseases. Here we have provided structural evidence showing that the expression of $\alpha 2-6$-sialylated $\mathrm{N}$-glycans varies depending on the differentiation potential of stem cells. Rapid, sensitive, and nondestructive process analytical testing (PAT) is essential for the stable supply of hMSCs, which could be used for monitoring during the cell production process. The quality control system to assess hMSCs for use as CTPs will become increasingly more important and stringent from the viewpoint of regulatory science.

Acknowledgments We thank Ms. Sayoko Saito for technical assistance with cell cultures and Dr. Akihiro Umezawa from the National Research Institute for Child Health and Development for providing Yub621c. The human iPS cell line 201B7 (HPS0063) was obtained from the RIKEN Bioresource Center. This work was supported in part by the "Development of Cell Manufacturing and Processing Systems for Commercialization of Regenerative Medicine" project from the Japan Agency for Medical Research and Development (AMED).

Open Access This article is distributed under the terms of the Creative Commons Attribution 4.0 International License (http:// creativecommons.org/licenses/by/4.0/), which permits unrestricted use, distribution, and reproduction in any medium, provided you give appropriate credit to the original author(s) and the source, provide a link to the Creative Commons license, and indicate if changes were made.

\section{References}

1. Friedenstein A.J., Chailakhyan R.K., Latsinik N.V., Panasyuk A.F., Keiliss-Borok I.V.: Stromal cells responsible for transferring the microenvironment of the hemopoietic tissues. Cloning in vitro and retransplantation in vivo. Transplant. 17(4), 331-340 (1974)

2. Friedenstein A.J., Petrakova K.V., Kurolesova A.I., Frolova G.P.: Heterotopic of bone marrow. Analysis of precursor cells for osteogenic and hematopoietic tissues. Transplant. 6(2), 230-247 (1968)

3. Zou J.P., Huang S., Peng Y., Liu H.W., Cheng B., Fu X.B., Xiang X.F.: Mesenchymal stem cells/multipotent mesenchymal stromal cells (MSCs): potential role in healing cutaneous chronic wounds. Int J Low Extrem Wounds. 11(4), 244-253 (2012). doi:10.1177/ 1534734612463935

4. Pittenger M.F., Mackay A.M., Beck S.C., Jaiswal R.K., Douglas R., Mosca J.D., Moorman M.A., Simonetti D.W., Craig S., Marshak D.R.: Multilineage potential of adult human mesenchymal stem cells. Science. 284(5411), 143-147 (1999)

5. Abdi R., Fiorina P., Adra C.N., Atkinson M., Sayegh M.H.: Immunomodulation by mesenchymal stem cells: a potential therapeutic strategy for type 1 diabetes. Diabetes. 57(7), 17591767 (2008). doi:10.2337/db08-0180

6. Giordano A., Galderisi U., Marino I.R.: From the laboratory bench to the patient's bedside: an update on clinical trials with mesenchymal stem cells. J. Cell. Physiol. 211(1), 27-35 (2007). doi:10.1002/ jсp.20959

7. Marquez-Curtis L.A., Janowska-Wieczorek A., McGann L.E., Elliott J.A.: Mesenchymal stromal cells derived from various tissues: biological, clinical and cryopreservation aspects. Cryobiology. 71(2), 181-197 (2015). doi:10.1016/j.cryobiol.2015.07.003

8. Tateno, H., Saito, S., Hiemori, K., Kiyoi, K., Hasehira, K., Toyoda, M., Onuma, Y., Ito, Y., Akutsu, H., Hirabayashi, J.: alpha26sialylation is a marker of the differentiation potential of human mesenchymal stem cells. Glycobiology (2016). doi:10.1093/ glycob/cww039

9. Takasaki S., Mizuochi T., Kobata A.: Hydrazinolysis of asparaginelinked sugar chains to produce free oligosaccharides. Methods Enzymol. 83, 263-268 (1982)

10. Kawashima H., Murata T., Yamamoto K., Tateishi A., Irimura T., Osawa T.: A simple method for the release of asparagine-linked oligosaccharides from a glycoprotein purified by SDS-polyacrylamide gel electrophoresis. J. Biochem. 111(5), 620-622 (1992)

11. Iwase H., Ishii-Karakasa I., Fujii E., Hotta K., Hiki Y., Kobayashi Y.: Analysis of glycoform of O-glycan from human myeloma immunoglobulin A1 by gas-phase hydrazinolysis following pyridylamination of oligosaccharides. Anal. Biochem. 206(1), 202-205 (1992)

12. Hase S.: High-performance liquid chromatography of pyridylaminated saccharides. Methods Enzymol. 230, 225-237 (1994)

13. Hase S., Ikenaka T., Matsushima Y.: Structure analyses of oligosaccharides by tagging of the reducing end sugars with a fluorescent compound. Biochem. Biophys. Res. Commun. 85(1), 257-263 (1978)

14. Fujimoto I., Menon K.K., Otake Y., Tanaka F., Wada H., Takahashi H., Tsuji S., Natsuka S., Nakakita S., Hase S., Ikenaka K.: Systematic analysis of $\mathrm{N}$-linked sugar chains from whole tissue employing partial automation. Anal. Biochem. 267(2), 336-343 (1999)

15. Tokugawa K., Oguri S., Takeuchi M.: Large scale preparation of PA-oligosaccharides from glycoproteins using an improved extraction method. Glycoconj. J. 13(4), iv (1996)

16. Natsuka S., Adachi J., Kawaguchi M., Ichikawa A., Ikura K.: Method for purification of fluorescence-labeled oligosaccharides by pyridylamination. Biosci. Biotechnol. Biochem. 66(5), 11741175 (2002). doi:10.1271/bbb.66.1174

17. Hasehira K., Tateno H., Onuma Y., Ito Y., Asashima M., Hirabayashi J.: Structural and quantitative evidence for dynamic glycome shift on production of induced pluripotent stem cells. Mol. Cell. Proteomics. 11(12), 1913-1923 (2012). doi:10.1074/ mcp.M112.020586

18. Wang Y.C., Stein J.W., Lynch C.L., Tran H.T., Lee C.Y., Coleman R., Hatch A., Antontsev V.G., Chy H.S., O'Brien C.M., Murthy S.K., Laslett A.L., Peterson S.E., Loring J.F.: Glycosyltransferase ST6GAL1 contributes to the regulation of pluripotency in human pluripotent stem cells. Sci. Rep. 5, 13317 (2015). doi:10.1038/srep13317

19. Alisson-Silva F., de Carvalho Rodrigues D., Vairo L., Asensi K.D., Vasconcelos-dos-Santos A., Mantuano N.R., Dias W.B., Rondinelli E., Goldenberg R.C., Urmenyi T.P., Todeschini A.R.: Evidences for the involvement of cell surface glycans in stem cell pluripotency and differentiation. Glycobiology. 24(5), 458-468 (2014). doi:10. 1093/glycob/cwu012

20. Swindall A.F., Londono-Joshi A.I., Schultz M.J., Fineberg N., Buchsbaum D.J., Bellis S.L.: ST6Gal-I protein expression is upregulated in human epithelial tumors and correlates with stem cell markers in normal tissues and colon cancer cell lines. Cancer Res. 73(7), 2368-2378 (2013). doi:10.1158/0008-5472.CAN-12-3424

21. Gu J., Isaji T., Sato Y., Kariya Y., Fukuda T.: Importance of Nglycosylation on alpha5betal integrin for its biological functions. Biol. Pharm. Bull. 32(5), 780-785 (2009) 